\title{
Cushing syndrome due to ritonavir-fluticasone interaction
}

\author{
Eduardo Canalejo MD, María S. Pacheco MD
}

Competing interests: Eduardo Canalejo has received payment from Merck Sharp \& Dohme Spain, as well as Abbott Laboratories for the development of educational presentations. No competing interests declared by María Pacheco.

This article has been peer reviewed.

Affiliations: From the Department of Internal Medicine (Canalejo); and the Department of Clinical Laboratory (Pacheco), Fuenlabrada University Hospital, Fuenlabrada (Madrid), Spain

\section{Correspondence to:} Eduardo Canalejo

Castrillero, ecanalejo@gmail.com

CMAJ 2012. DOI:10.1503 /cmaj.111315

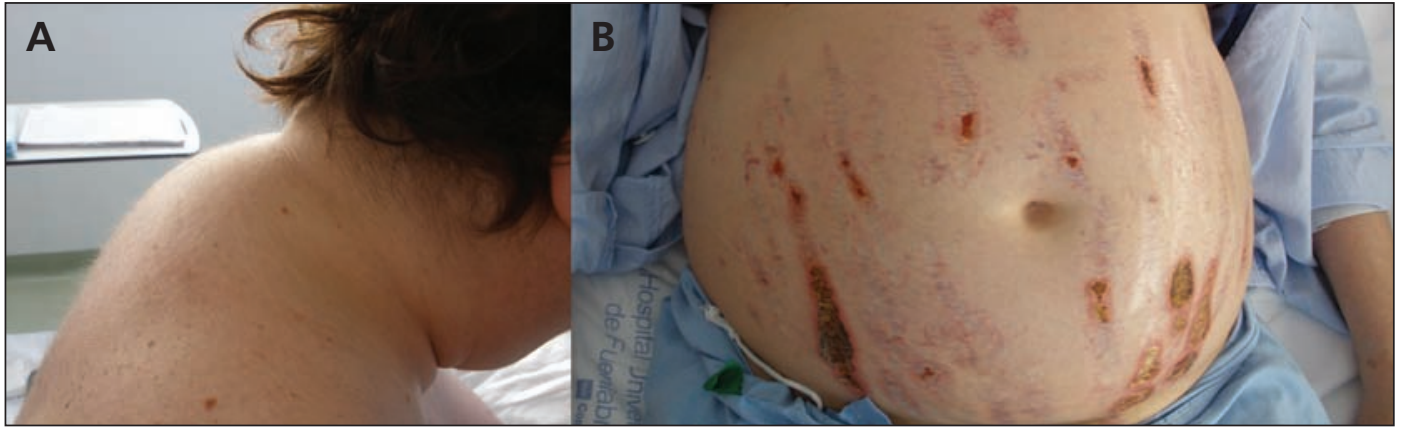

Figure 1: Dorsocervical fat pad (A) and prominent abdominal striae with secondary impetiginization (B) in a 36-year-old woman with iatrogenic Cushing syndrome.

A 36-year-old woman with HIV presented with recent weight gain, marked abdominal striae and proximal muscle weakness. She had a four-year history of type 2 diabetes mellitus that had been well controlled until recently by metformin and insulin glargine, and she had longstanding asthma that had been treated for the past two years with inhaled fluticasone-salmeterol. After one year of antiretroviral therapy with tenofovir, emtricitabine and efavirenz for treatment of HIV, her medication was switched to a ritonavircontaining regimen (atazanavir-ritonavir) because of virologic failure. Within three months, she developed proximal myopathy, increased abdominal girth, multiple abdominal striae and mild leg edema. She reported the onset of poor diabetes control, amenorrhea and weight gain of $8 \mathrm{~kg}$.

On examination, she had cushingoid facies, dorsocervical fat pad, central obesity and prominent abdominal striae (Figure 1). Her morning serum cortisol level was low (25 [normal 118$618 \mathrm{nmol} / \mathrm{L}$ ) as was her adrenocorticotropic hormone level (1.3 [normal 1.8-10.1] pmol/L), which was consistent with adrenal suppression.

The patient was given a diagnosis of Cushing syndrome with adrenal suppression by exogenous glucocorticoids caused by the interaction between fluticasone and ritonavir. Fluticasone was stopped. One week later, mild symptoms of hypocortisolism (fatigue, nausea, postural hypotension) developed, which resolved without the need for glucocorticoid replacement therapy. One month later, her early morning cortisol level normalized, and glycemic control was restored. The cushingoid features completely resolved within four months.

Ritonavir, a potent inhibitor of cytochrome P450-3A4 (CYP3A4), is commonly used in low doses to boost plasma levels of other protease inhibitors in patients with HIV. Fluticasone, a potent glucocorticoid, is rapidly metabolized by CYP3A4 and has minimal systemic effects at recommended dosages. However, coadministration of ritonavir with either inhaled or intranasal fluticasone results in increased serum concentrations of fluticasone and can lead to systemic complications. ${ }^{1,2}$ Other inhaled glucocorticoids, such as beclomethasone and budesonide, appear to be safer options because of their lower binding affinity for glucocorticoid receptors and shorter elimination half-life. ${ }^{1}$ However, they are also CYP3A4 substrates, and similar cases have been described. ${ }^{3}$ Caution should be used when any inhaled glucocorticoid is combined with ritonavir.

\section{References}

1. Foisy MM, Yakiwchuk EMK, Chiu I, et al. Adrenal suppression and Cushing's syndrome secondary to an interaction between ritonavir and fluticasone: a review of the literature. HIV Med 2008;9:389-96.

2. Valin N, De Castro N, Garrait V, et al. Iatrogenic Cushing's syndrome in HIV-infected patients receiving ritonavir and inhaled fluticasone: description of 4 new cases and review of the literature. J Int Assoc Physicians AIDS Care (Chic) 2009;8: 113-21.

3. Kedem E, Shahar E, Hassoun G, et al. Iatrogenic Cushing's syndrome due to coadministration of ritonavir and inhaled budesonide in an asthmatic human immunodeficiency virus infected patient. J Asthma 2010;47:830-1. 